\title{
Comparative Study on Adolescents' Response to Parents' Marital Conflict in Japan and China
}

\author{
Xinhe Zhang ${ }^{1)}$ \\ ${ }^{1)}$ Graduate School of Education, Tohoku University
}

\begin{abstract}
The Adolescents' Response to Parents' Marital Conflict Scale was developed and administered to 602 high-school students in the $1 \mathrm{st}, 2 \mathrm{nd}$, and 3 rd grade in order to examine its constructs and internal consistency. A multiple-group confirmatory factor analysis showed that the scale had a common structure across the Japanese and Chinese samples. To some extent, the internal consistency of the Adolescents' Response to Parents' Marital Conflict Scale was confirmed. When encountering a parents' marital conflict, women scored higher than men on "depression/anxiety," "irritation/anger," and "interventional behavior" in both countries. Moreover, 3rd grade (high-school) students scored higher on "parents' conflict resolution" than 1st and 2nd grade students in both countries. There were some differences between the two countries in the adolescents' response: Japanese adolescents scored high on “coping efficacy toward self," and "avoidance behavior," and Chinese adolescents scored high on "parents' conflict resolution," "depression/anxiety," "irritation/anger," and “interventional behavior." The differences of adolescents' response between the two countries were discussed from the perspective of cross-cultural psychology.
\end{abstract}

KEY WORDS: Adolescents' Response to Parents' Marital Conflict Scale, Chinese adolescents, Japanese adolescent.

\section{Introduction}

Researches on young adolescents have indicated the association between parents' marital conflicts and internalizing problems (Amato \& Afifi, 2006; Davis \& Windle, 2001). The cognitive-contextual framework proposed by Grych and Fincham (1990), and the emotional security hypothesis proposed by Davis and Cummings (1994) have drawn attention for interpreting the association between parents' marital conflicts and

Correspondence to ZhANG, Graduate School of Education, Tohoku University, 27-1 Kawauchi, Aoba-ku, Sendai-city, 980-8576, Japan.

E-mail: guhong15_qiaoxinka@yahoo.co.jp children's psychological adjustment. Grych and Fincham (1993) focused on the framework of children's cognition of parents' marital conflicts, claiming that the cognition of the "conflict properties," "threat," and "self-blame" is the intermediary mechanism that underlies the association between children's internalizing and externalizing problems and parents' marital conflicts. Davis and Cummings (1994) have proposed an intermediary mechanism in which children's psychological adjustment is influenced by parents' marital conflicts through the threats on that child's or the family' $\mathrm{s}$ emotional security. Davis and Cummings examined the association between parents' marital conflicts and children's internalizing and externalizing problems by estimating 
emotional and standardized based on the Japanese and interventional/avoidance behavior toward Chinese social systems and cultural parents' marital conflicts as the indexes of emotional security. However, Turner and Barret (1998) have claimed that both the emotional security hypothesis and the cognitive-contextual framework are important in the process of explaining children's adjustment, indicating that both children's cognition of parents' marital conflicts and emotional security toward their parents have a unique impact on children's adjustment. This suggests that it is important to consider not only cognitive response but also emotional and behavioral responses of children.

A number of studies related to the influence of parents' marital conflicts on children's adjustment have been conducted in the Western countries, while the number of empirical research on this area has been limited in Japan and China. (Yamamoto \& Ito, 2012; Chi, 2008). The existing scales for examining children's perspective on parents' marital conflicts have been established mainly for the context of the Western population. Thus they have not been backgrounds.

This study aimed to develop a scale to investigate adolescents' response toward parents' marital conflict and to examine its constructs, and internal consistency in Japan and China. Upon verifying the homogeneity of the data and the constructs of the scale, an international comparison was made by weighing the subscale scores of the adolescents' response to parents' marital conflict in Japan and in China.

\section{Methods}

Procedures

A questionnaire survey was conducted separately in September 2012 and November 2012 at a private high school located in the Tohoku District of Japan and at a public high school located in Northern China. 602 high-school students in the 1st, 2nd, and 3rd grade participated in this study. The participants without parents (including those with single parents), those with 5 or more

Table.1 sample description

\begin{tabular}{|c|c|c|c|c|c|c|c|c|c|}
\hline & & \multicolumn{4}{|c|}{ Japan } & \multicolumn{4}{|c|}{ China } \\
\hline & & \multicolumn{8}{|c|}{ grade } \\
\hline & & $1 \mathrm{st}$ & $2 \mathrm{nd}$ & $3 \mathrm{rd}$ & sum & $1 \mathrm{st}$ & 2nd & $3 \mathrm{rd}$ & sum \\
\hline & male & 29 & 32 & 50 & 111 & 18 & 80 & 19 & 117 \\
\hline & female & 43 & 53 & 44 & 140 & 30 & 95 & 23 & 148 \\
\hline & missing & 0 & 0 & 0 & 0 & 2 & 9 & 0 & 11 \\
\hline & sum & 72 & 85 & 94 & 251 & 50 & 184 & 42 & 276 \\
\hline
\end{tabular}


missing items in their responses, or those marked "I could not imagine at all" on the check item involving scene imagination were removed from the analysis. The total number of valid responses were 527 (251 Japanese and 276 Chinese) (Table 1). The average age of the participants was $16.73(S D=0.82)$.

\section{Measures}

A Chinese version of the Adolescents' Response to Parents' Marital Conflict Scale was first created, which was later translated in to Japanese version. The author independently performed the basic translation, and then requested three Chinese students majoring in Psychology to perform a back-translation. Afterwards, the scale was revised for fluency, based on the opinions of Japanese graduate students majoring in Psychology.

\section{Face sheet}

Participants were asked to fill up the basic demographics (gender, age, and grade-level) of themselves and their family members.

Adolescents' Response to Parents' Marital Conflict Scale

Participants were asked to imagine the described parents' marital conflict situation it would as actually be happening in their family and provide answers for the Adolescents' Response to Parents' Marital Conflict Scale. In the current research, we focused on the parents' marital conflict situation caused by household chaos. Items related to cognitive response: according to the CPIC (Children's Perception of Interparental Conflict Scale) by Grych et al. (1992), and other previous studies (Chi, 2008), three constructs for "parental factors" and three constructs for "child factors" were proposed, and a total of 24 items were developed. Items related to emotional response: referring to previous studies on psychological stress responses of high school students (Sakano et al., 1994; Zheng, 2001), two constructs, "irritation/anger" and "depression/anxiety" were proposed, and a total of 10 items were created. Items related to behavioral response: referring to previous studies on children's coping strategies and parents' marital conflicts (Grych et al., 1993; Cummings et al., 1994; Xiao \& Li, 2010), it was hypothesized two constructs for "interventional behavior" and "avoidance behavior," and developed 11 items. Check items: 4 items were assumed, including the item asking how well the participant was able to imagine the described scene. These items were evaluated on a 4-point scale.

\section{Results}

Exploratory factor analysis by country

After confirming the floor and ceiling effects, an exploratory factor analysis using the principal factor method and promax rotation was performed on the Japanese and Chinese responses. The results displayed similarity in factor structure but a difference in item structure between Japanese and Chinese participants. In both samples, two factors for cognitive response involving "parental factors" ("threat about parents" and "parents' conflict resolution"), two factors for cognitive response involving "child factors" ("threat about oneself" and "coping efficacy toward self"), two factors for emotional response ("irritation/anger" and 
"depression/anxiety"), and two factors for behavioral response ("interventional behavior" and "avoidance behavior") were extracted. The internal consistency of the subscales was $\alpha$ $=.54-.89$. While the "coping efficacy toward self" among cognitive response involving "child factors" was relatively low in Chinese sample $(\alpha=.54)$, others showed .62 or higher.

\section{Exploratory factor analysis using all data}

The Japanese and Chinese data were combined, confirming the floor and ceiling effects, an exploratory analysis using the principal factor method and promax rotation was performed. The results displayed similar factor structure with the results of the exploratory factor analysis conducted by country, but showed a difference in item structure. The internal consistency of the subscales was $\alpha=.59-.85$ using the combined data. While the "coping efficacy toward self" among cognitive response involving "child factors" was relatively low $(\alpha=.59)$, others showed .73 or higher. The scale was created based on both the results from the exploratory factor analysis by country and from the combined data, while taking the content of each factor and the items into consideration. The scale employed 33 items including: 31 items that were common among the Japanese, Chinese, and the combined data; one item that was common among the Japanese and the combined data; and one item that was common among the Chinese and the combined data.

Examining the factor structure and the internal consistency

A confirmatory factor analysis was performed to examine the homogeneity of the Japanese and Chinese samples for the Adolescents' Response to Parents' Marital Conflict Scale. First, the data was analyzed by country. The results are shown in Table 2 . From these results, it was indicated that the compatibilities of the model with the data were reasonable among all of the subscales. The models did not fit the data well with the Japanese data, yet the study proceeded to examine the factorial invariance.

Next, in order to examine the factorial invariance, a multiple-group confirmatory factor analysis was conducted, and the following three models were compared and examined; the pattern invariant model (model 0 ), the measurement invariant model (model 1), and the strong factorial invariant model (model

Table. 2 The results of confirmatory factor analysis by country

\begin{tabular}{lllllll}
\hline & \multicolumn{3}{c}{ Japan } & \multicolumn{3}{c}{ China } \\
\cline { 2 - 7 } & GFI & AGFI & RMSEA & GFI & AGFI & RMSEA \\
\hline Cognitive response(parental) & .92 & .87 & .10 & .96 & .93 & .06 \\
Cognitive response(child) & .90 & .83 & .11 & .95 & .92 & .07 \\
Emotional response & .96 & .91 & .09 & .96 & .91 & .09 \\
Behavioral response & .90 & .81 & .13 & .96 & .93 & .06 \\
\hline
\end{tabular}


2). The results are shown in Table 3. The results suggested a high possibility that both populations from Japan and China had the same factor structure for Adolescents' Response to Parents' Marital Conflict.

The RMSEA values of confirmatory factor analysis by country and multiple-group confirmatory factor analysis were relatively high. Theoretically, there is a close fit if the RMSEA is less than 0.05 , whereas a value of RMSEA greater than 0.1 indicates a poor fit. An RMSEA in the range of 0.05 to 0.1 is considered an indication of fair fit. Although the model was preferentially selected according to the author's assumption in this study, it is expected that stable results can be obtained as data increase in further research.

The internal consistency of the subscales was $\alpha=.59-.86$. While the "coping efficacy toward self" among cognitive response involving "child factors" was relatively low $(\alpha=.59)$, others showed .70 or higher. Therefore, to some extent the reliability of the Adolescents' Response to Parents' Marital Conflict Scale was confirmed.

\section{Comparing Japanese and Chinese scores}

A three-way factorial ANOVA of "country," "gender," and "grade-level" $(2 \times 2 \times 3)$ was performed on the 8 subscale responses from the Japanese and Chinese participants.

The main effect of "country" was significant in a total of six factors; "parents' conflict resolution", $F_{(1,504)}=55.24, p<.001$; "coping efficacy toward self", $F_{(1,504)}=28.12, p<.001$; "depression/anxiety", $F_{(1,504)}=28.72, p<.001$; "irritation/anger ", $F_{(1,504)}=3.91, p<.05$; "interventional behavior", $F_{(1,504)}=236.84, p$ $<.001$; and "avoidance behavior ", $F_{(1,504)}=$ 164.06, $p<.001$. Japanese adolescents scored high on "coping efficacy toward self," and "avoidance behavior," and Chinese adolescents scored high on "parents' conflict resolution," "depression/anxiety," “irritation/anger," and "interventional behavior."

There was main effect of "gender" on “depression/anxiety", $F_{(1,504)}=8.12, \quad p<.01$; "irritation/anger", $F_{(1,504)}=7.83, p<.01$; and "interventional behavior", $F_{(1,504)}=4.98, p$ $<.05$. Women scored higher on "interventional behavior" in both countries.

Table. 3 The results of multiple-group confirmatory factor analysis

\begin{tabular}{lllllll}
\hline & model & GFI & AGFI & RMSEA & AIC & BCC \\
\hline Cognitive response & model 0 & .94 & .90 & .06 & 212.81 & 215.83 \\
(parental ) & model 1 & .93 & .90 & .06 & 219.02 & 221.48 \\
& model 2 & .90 & .90 & .06 & 217.09 & 219.47 \\
\hline Cognitive response & model 0 & .93 & .88 & .07 & 246.58 & 249.60 \\
(child) & model 1 & .93 & .89 & .06 & 236.46 & 238.92 \\
& model 2 & .92 & .89 & .06 & 241.83 & 244.21 \\
\hline \multirow{3}{*}{ Emotional response } & model 0 & .96 & .91 & .06 & 140.68 & 142.57 \\
& model 1 & .95 & .91 & .06 & 138.08 & 139.65 \\
& model 2 & .95 & .92 & .06 & 136.61 & 138.13 \\
\hline \multirow{3}{*}{ Behavioral response } & model 0 & .93 & .87 & .07 & 207.16 & 219.96 \\
& model 1 & .92 & .87 & .07 & 219.74 & 221.73 \\
& model 2 & .92 & .88 & .07 & 218.04 & 219.96 \\
\hline
\end{tabular}


"depression/anxiety," "irritation/anger," and

The main effect of "grade-level" was found only in "parents' conflict resolution", $F_{(2,504)}=$ $5.20, p<.01)$. The score of the 3rd grade students was significantly higher than that of the 1 st grade and the 2 nd grade students. No significant differences were found between 2 nd grade students and 1st grade students.

The interaction between "country" and "grade-level" was only significant on "depression/anxiety", $F_{(2,504)}=4.35, p<.05$. Chinese adolescents in the 1st and 2nd grade scored higher than Japanese adolescents of the same grade-level, but there was no significant difference between 3rd grade Japanese and Chinese adolescents.

\section{Discussions}

The results of this study showed several commonalities between Japanese and Chinese adolescences. Firstly, for the populations of both countries, the measurement invariance of the constructive concept of Adolescents' Response to Parents' Marital Conflict were confirmed, suggesting the high possibility of having the same factor structure. In other words, “Adolescents' Response to Parents' Marital Conflict" is a relatively stable construct in Japan and China. Secondly, when facing parents' marital conflict, women, in both countries, are more likely to show stronger emotional response such as depression, anxiety, irritation, and anger, and are more likely to intervene with the conflict scene than men. Moreover, 3rd grade (high-school) students, in both countries, are more likely to predict the parents' marital conflict to end in reconciliation, than 1 st and 2 nd grade students. This result indicates a certain pattern of Adolescents' Response to Parents' Marital Conflict associated with the differences in sex and a developmental stage, regardless of the cultural differences.

Furthermore, the assessment suggested several differences in the response of Japanese and Chinese adolescences. When facing parents' marital conflict, Japanese adolescents were more likely to feel that they could stay calm and to avoid conflict scene than Chinese adolescents. On the other hand, Chinese adolescents showed stronger emotional response and were found to be more likely to intervene with the conflict scene than Japanese adolescents, even if their parents' conflict was predicted to eventually reconcile. According to the psychological stress model, Chinese adolescents might be more sensitive to perceiving parents' marital conflict as a stress factor than Japanese adolescents. Many couples have been trying to maintain their relationship for the sake of their children's growth in a complete family since China launched the one-child policy. However, it results in more frequent exposure of children in parents' marital conflicts. Chinese adolescents are more likely to take responsibility for solving their parents' conflicts, for they view that their parents are staying together only for their sake. While in Japan, parents' marital conflicts are usually considered as the problems between couples in which children are not involved too much. Presumably, there are differences 
between the two countries in adolescents' coping strategies of parents' marital conflicts, based on cultural factors. There have been other research, as well, asserting that the selections on 'conflict resolution strategies' are pertinent to cultural values (Ting-Toomey, 1994). Although both Japan and China are regarded as collective culture, as with the coping strategies in conflict situation, Chinese adolescents are more likely to intervene in their parents' marital conflicts which are more like to occur in individualistic culture.

By shedding light on the characteristics of adolescents in Japan and China, this study provides additional clues as to how to protect adolescents from parents' marital conflicts.

\section{References}

Amato, P. R., \& Tamara, D. A. (2006). Feeling Caught Between Parents: Adult Children's Relations With Parents and Subjective Well-Being. Journal of Marriage and Family, $68,222-235$.

Chi, L., \& Yu, G. (2008). Cognitive Appraisals as a Mediator in the Association Between Marital Conflict and Adolescents' Self-esteem. Psychological Science, 31, 1069-1073.

Davis, P. T., \& Cummings, E. M. (1994). Marital Conflict and Child Adjustment: An Emotional Security Hypothesis. Psychological Bulletin, 116, 387-411.

Davis, P. T., \& Windle, M. (2001). Interparental Discord and Adolescent Adjustment Trajectories: The Potentiating and Protective Role of Intrapersonal
Attributes. Child Development, 72, 1163-1178.

Grych, J. H., \& Fincham, F. D. (1990). Marital Conflict and Children's Adjustment : A Cognitive-Contextual Framework. Psychological Bulletin, 108, 267-290.

Grych, J. H., \& Fincham, F. D. (1993). Children's Appraisals of Marital Conflict : Initial Investigations of the Cognitive-contextual Framework. Child Development, 71, 1648-1661.

Grych, J. H., Seid, M., \& Fincham, F. D. (1992). Assessing Marital Conflict from the Child's Perspective: The Children's Perception of Interparental Conflict Scale. Child Development, 63, 558-572.

Sakano, Y., Shimada H., Miura M., Mori H., Oda M., \& Saruwatari S. (1994). The Effect of Individual Differences in Cognitive Responses on Psychological Stress Responses in Senior High School Students. Human Science, 7, 75-90.

Ting-Toomey, S. (1994). Managing conflict in intimate intercultural relationships. In D. Cahn(Ed.), Conflict in personal relationships (pp. 47-77). Hillsdale, N. J: L. Erlbaum.

Turner, C. M., \& Barrett, P. M. (1998). Adolescent Adjustment to Perceived Marital Conflict. Journal of Child and Family Studies, 7, 499-513.

Xiao Q., \& Li D. (2010). The Coping Strategies to Parental Conflicts of Students in Junior Middle School. Psychological Science, 33, 959-962.

Yamamoto S., \& Ito Y. (2012). Relationship between Parents' Marital Conflicts Witnessed 
by Adolescents and their Psychological Health. Japanese Journal of Family Psychology, 26, 83-94.

Zheng Q., Chen S., Zheng S., \& Huang L. (2001). A Preliminary Study of the Stress of Middle School Students. Psychological Science, 24, 212-213. 